\section{De la mesure en toute chose: dérégulation de p53, cancer et syndromes télomériques}

Sara Jaber, Iva Simeonova, Franck Toledo

\author{
${ }^{\mathrm{l} G e ́ n e ́ t i q u e ~ d e ~ l a ~ s u p p r e s s i o n ~ t u m o r a l e, ~}$ \\ Institut Curie, centre de recherche, \\ CNRS UMR 3244, UPMC Université Paris 6, \\ 26, rue d'Ulm, 75248 Paris Cedex 05, France. \\ franck.toledo@curie.fr
}

Ces observations semblaient particulièrement importantes puisque chez l'homme la mutation de Dkcl, Rtell ou Tinf2 peut causer une dyskératose congénitale, alors que Terfl a été impliqué dans l'aplasie médullaire. En utilisant des souris $p 53^{\Delta 31 / \Delta 31}$ de fonds génétiques différents, nous avons également observé qu'un niveau plus élevé de Rtell permet d'atténuer la sévérité des symptômes des souris atteintes. Le phénotype télomérique causé par la délétion du CTD dépend donc des niveaux d'expression d'au moins l'un des gènes télomériques régulés par $p 53$.

La suractivation de $\mathrm{p} 53$ peut-elle causer une dyskératose congénitale chez l'homme?

Nos résultats chez la souris posent évidemment la question de leur pertinence en santé humaine. À ce titre, il est tout d'abord important de constater que les domaines carboxy-terminaux des protéines p53 murine et humaine sont très conservés. Par ailleurs, nous avons ciblé la mutation codant $p 53^{\Delta 31}$, ainsi que trois autres mutations non-sens codant respectivement $p 53^{\Delta 36}, p 53^{\Delta 45}$ ou $p 53^{\Delta 52}$, dans des fibroblastes embryonnaires murins par recombinaison homologue de type Cre/Lox. De ces quatre mutations affectant l'exon 10 du gène $p 53$ murin, seules les deux dernières entraînent des niveaux plus faibles d'expression et d'activité de p53. Or les mutations nonsens équivalentes recensées dans les cancers humains codent également pour une p53 tronquée d'au moins 45 acides aminés [4]. Par ailleurs, une fraction importante des patients atteints de dyskératose congénitale (ou de son 


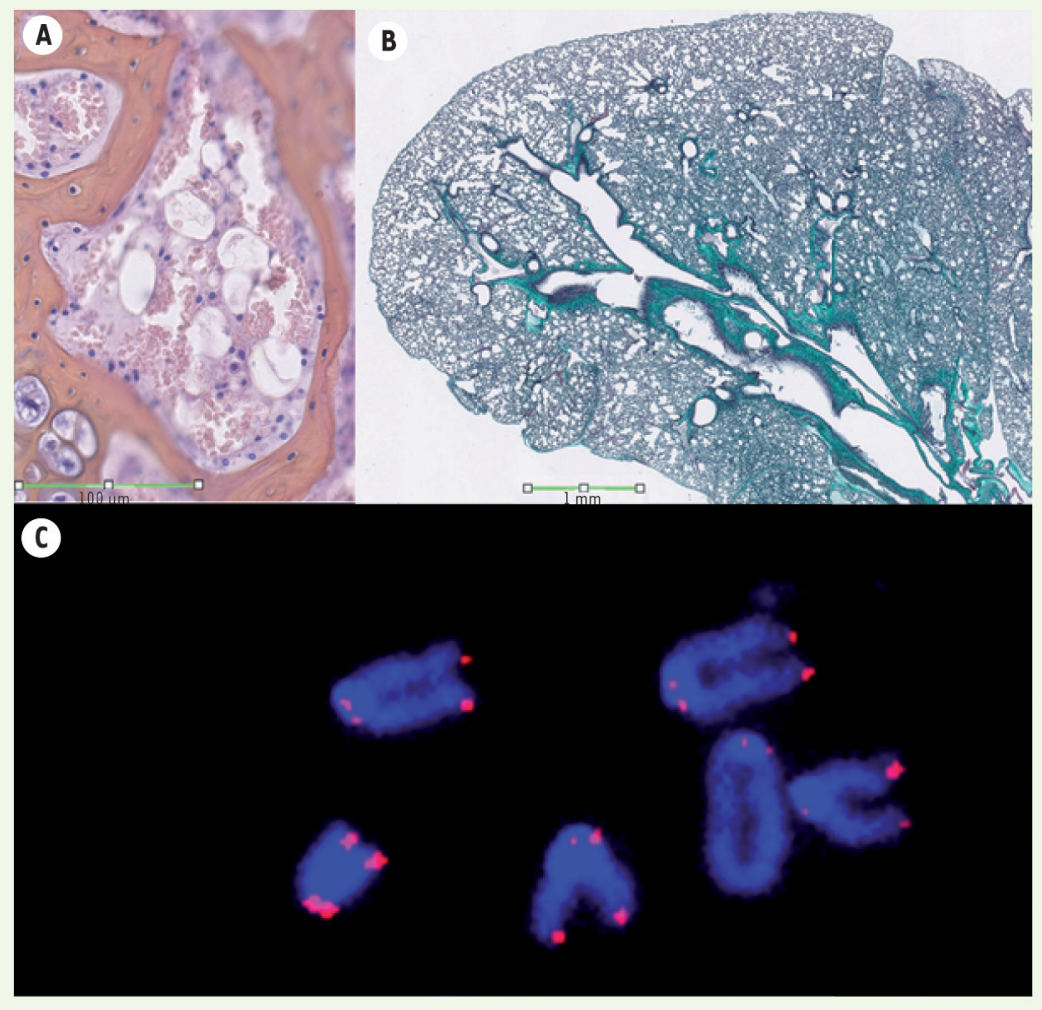

variant sévère le syndrome d'HoyeraalHreidarsson [6]) ont des mutations dans les gènes $D K C l$ ou RTELl, et nous avons montré que, comme chez la souris, l'activation de la p53 humaine entraîne une diminution de l'expression de ces deux gènes.

Étant donné que les mutations des gènes dont l'implication dans une dyskératose congénitale a été identifiée n'expliquent que $50 \%$ des cas cliniques, il devrait être intéressant de rechercher des mutations du gène TP53 chez les patients souffrant d'un syndrome télomérique dont l'origine moléculaire reste inexpliquée.

Importance d'un dosage précis de p53 Outre sa fonction bien connue de suppresseur de tumeurs, p53 intervient dans des processus très variés tels que la longévité, la fertilité et la production de cellules souches. P53 est également impliquée dans certaines maladies comme le diabète et différentes maladies neurodégénératives $[7,8](\rightarrow)$.

$(\rightarrow)$ Voir la Synthèse de M. Lacroix et al., page 1125 de ce numéro
Ces multiples fonctions justifient l'existence d'une régulation précise de $p 53$ via - entre autres - ses principaux régu-

De fait, plusieurs modèles murins ont révélé l'impact de variations des niveaux d'activité de p53. En l'absence de p53, toutes les souris développent des tumeurs, fatales en moins de six mois [9]. À l'inverse, chez les mutants $\mathrm{Mdm}^{-/-}$ou $M \mathrm{dm}^{-/-}$, l'incapacité d'inhiber $\mathrm{p} 53$ se traduit par une létalité embryonnaire [10]. Entre ces deux extrêmes, différents phénotypes intermédiaires ont été observés. Par exemple, la plupart des souris exprimant des p53 mutantes dont l'activité est réduite, mais non nulle ( $p 53^{R 172 P}$ ou $p 53^{\Delta P}$ ), développent des tumeurs dans la deuxième année de leur vie $[11,12]$. Les souris Super-p53, dont l'activité de p53 est une fois et demi supérieure à la normale, développent moins de tumeurs que les souris sauvages [13]. Des souris $p 53^{+/ m}$, exprimant une p53 mutante avec une activité un peu plus forte, sont également lateurs négatifs $\mathrm{Mdm} 2$ et $\mathrm{Mdm} 4$.
Figure 1. Les souris exprimant la $p 53^{\Delta 31}$ présentent des caractéristiques de dyskératose congénitale. A. Marquage hématoxyline et éosine d'une section de sternum. La rareté des cellules (en bleu) dans la moelle osseuse de ces souris indique un défaut d'hématopoïèse. B. Marquage au trichrome de Masson d'une section de poumon. La coloration intense du collagène (en vert) démontre une fibrose pulmonaire. C. Analyse de la longueur des télomères par FISH quantitative avec une sonde spécifique des télomères (en rouge). La faible intensité du signal indique que les chromosomes des cellules mutantes ont des télomères anormalement courts.

résistantes au développement tumoral, mais souffrent d'un vieillissement accéléré [14]. Notre étude montre que dans les souris $p 53^{\Delta 31 / \Delta 31}$, une activité de p53 encore plus élevée conduit à un raccourcissement télomérique et à une aplasie médullaire, entraînant la mort avant l'âge adulte. II est important de noter que la plupart des souris $p 53^{+/ \Delta 31}$ (que nous avons observées pendant un an) ne présentent pas de phénotype notable, alors que les animaux $p 53^{+/ \Delta 31} \mathrm{Mdm}^{+/-}$présentent le même phénotype que les souris $p 53^{\Delta 31 / \Delta 31}$ [4]. Ces résultats révèlent qu'une variation de l'activité p53 d'un facteur deux est suffisante pour induire ou non une aplasie médullaire létale, ce qui démontre l'importance physiologique de la régulation très précise de $p 53$.

\section{Conclusion}

De la mesure en toute chose: cette maxime célèbre semble s'appliquer parfaitement à la régulation de $\mathrm{p} 53$. En effet, si une activité anormalement basse de p53 favorise l'apparition de cancers, une activité accrue peut conduire à un vieillissement accéléré, voire une létalité prépubère ou même embryonnaire. Nos résultats ont révélé que le contrôle du métabolisme télomérique est une fonction importante de $\mathrm{p} 53$, et qu'une régulation fine de cette fonction est nécessaire à la survie de l'animal. Ainsi, plus de 


\begin{tabular}{|c|c|}
\hline $\begin{array}{c}\text { Caractéristiques } \\
\text { phénotypiques des patients } \\
\text { atteints de DC }\end{array}$ & $\begin{array}{c}\text { Pourcentage } \\
\text { des souris } p 53^{\Delta 31 / \Delta 31} \\
\text { présentant ces caractéristiques }\end{array}$ \\
\hline \multicolumn{2}{|l|}{$\begin{array}{l}\text { Caractéristiques physiques } \\
\text { (triade diagnostique) }\end{array}$} \\
\hline $\begin{array}{l}\text { Hyperpigmentation cutanée } \\
\text { Dystrophie unguéale } \\
\text { Leucoplasie orale }\end{array}$ & $\begin{array}{l}100 \% \\
8 \% \\
100 \%\end{array}$ \\
\hline \multicolumn{2}{|l|}{ Symptômes pathologiques } \\
\hline $\begin{array}{l}\text { Aplasie médullaire } \\
\text { Fibrose pulmonaire }\end{array}$ & $\begin{array}{l}100 \% \\
87 \%\end{array}$ \\
\hline \multicolumn{2}{|l|}{ Phénotype moléculaire } \\
\hline Dysfonctionnement télomérique & $100 \%$ \\
\hline \multicolumn{2}{|l|}{$\begin{array}{l}\text { Autres phénotypes potentiellement } \\
\text { associés }\end{array}$} \\
\hline Hypertrophie cardiaque & $100 \%$ \\
\hline Taille réduite & $63 \%$ \\
\hline Atrophie testiculaire & 91 \% (des mâles) \\
\hline Hypoplasie cérébelleuse & $30 \%$ \\
\hline
\end{tabular}

Tableau I. Manifestations phénotypiques de la dyskératose congénitale (DC) chez les souris p53 $3^{\Delta 31 / \Delta 31}$.

30 ans après sa découverte, $p 53$ ne cesse de nous surprendre par l'étendue de ses fonctions, et par la variété des conséquences de sa dérégulation. $\diamond$

Moderation in all things: p53

deregulation, cancer and telomere

syndromes

\section{LIENS D'INTÉRÊT} concernant les données publiées dans cet article.

\section{RÉFÉRENCES}

1. Toledo F, Wahl GM. Regulating the p53 pathway: in vitro hypotheses, in vivo veritas. Nat Rev Cancer 2006 ; $6: 909-23$
Les auteurs déclarent n'avoir aucun lien d'intérêt

\section{LA FONDATION PREMUP : UN OPÉRATEUR} DE TERRAIN EN PÉRINATALITÉ RECONNU POUR SON EXCELLENCE ET SON INTERDISCIPLINARITÉ

a Fondation de coopération scientifique PremUp, unique en Europe, inte naissance, par la protection de la santé de la femme enceinte et du nouveau-né.

- Information et sensibilisation de la société civile,

- Recherche interdisciplinaire sur de grands projets prioritaires,

- Soins et formation des professionnels en périnatalité.

Reconnue d'utilité publique, la Fondation a été créée il y a cinq ans à l'initiative des Ministères de la Recherche et de la Santé.

Ses membres fondateurs en 2013 sont l'AP-HP, le CHI de Créteil, l'Inserm, l'IRD et les universités Paris Descartes, Paris Diderot, Pierre et Marie Curie et Paris Sud.

La Fondation regroupe un pôle de soins prenant en charge chaque année plus de 20.000 naissances (les Maternités et Services de néonatologie des hôpitaux Robert Debré, Antoine Béclère/Clamart, Armand Trousseau et Kremlin Bicêtre, du Centre Hospitalier Cochin Port-Royal et du Centre hospitalier intercommunal de Créteil) - et un pôle interdisciplinaire de 220 chercheurs coopérant sur la mère et l'enfant.

Grâce à son statut juridique particulier, cette structure d'utilité publique représente une passerelle entre le secteur public et le secteur privé et permet d'initier de grands projets de recherche interdisciplinaires sur la prématurité et le Retard de Croissance Intra Utérin notamment.

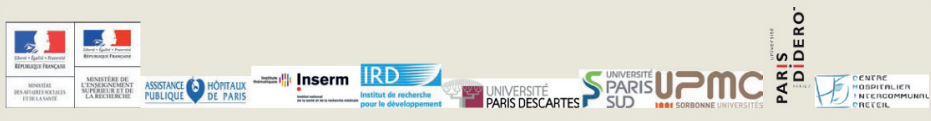

2. DP Lane. Cancer. p53, guardian of the genome. Nature $1992 ; 358: 15-6$

3. Foord OS, Bhattacharya P, Reich Z, Rotter V. A DNA binding domain is contained in the $\mathrm{C}$-terminus of wild type 533 protein. Nucleic Acids Res $1991 ; 19$ : 5191-8.

4. Simeonova I, Jaber S, Draskovic I, et al. Mutant mice lacking the $\mathrm{p} 53 \mathrm{C}$-terminal domain model telomere syndromes. Cell Rep 2013 ; 3 : 2046-58.

5. Hoareau-Aveilla C, Henry Y, Leblanc T. La dyskératose congénitale. Med Sci (Paris) $2008 ; 24: 390-8$.

6. Le Guen T, Jullien L, Schertzer M, et al. RTELl (regulator of telomere elongation helicase 1), une hélicase d'ADN essentielle à la stabilité du génome. Med Sci (Paris) $2013 ; 29$ : 1138-44.

7. Brady CA, Attardi LD. p53 at a glance. J Cell Sci 2010 ; $123: 2527-32$.

8. Lacroix M, Linares LK, Le Cam L. Rôle du suppresseur de tumeur $\mathrm{p} 53$ dans le contrôle du métabolisme. Med Sci (Paris) $2013 ; 29: 1125-30$.

9. Donehower LA, Harvey M, Slagle BL, et al. Mice deficient for $p 53$ are developmentally normal but susceptible to spontaneous tumours. Nature 1992 ; $356: 215-21$.

10. Marine JC, Francoz S, Maetens M, et al. Keeping p53 in check : essential and synergistic functions of $\mathrm{Mdm} 2$ and Mdm4. Cell Death Differ 2006 ; 13 : 927-34.

11. Liu G, Parant JM, Lang G, et al., Chromosome stability, in the absence of apoptosis, is critical for suppression of tumorigenesis in Trp53 mutant mice. Nat Genet $2004 ; 36: 63-8$.

12. Fang M, Simeonova I, Bardot B, et al. Mdm4 loss in mice expressing a 53 hypomorph alters tumor spectrum without improving survival. Oncogene, 2013; doi : 10.1038/onc.2013.62.2013

13. García-Cao I, García-Cao M, Martín-Caballero J, et al. EMBO J $2002 ; 21: 6225-35$.

14. Tyner SD, Venkatachalam S, Choi J, et al. p53 mutant mice that display early ageing-associated phenotypes. Nature 2002 ; 415 : 45-53.
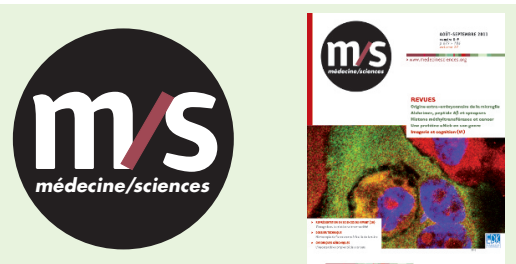

\section{Abonnez-vous \\ à médecine/sciences}

Bulletin d'abonnement page 1104 dans ce numéro de $\mathrm{m} / \mathrm{s}$ 